\title{
Imaging the Atherosclerosis with 64-Detector Row Computed Tomography in Homozygous Familial Hypercholesterolemia
}

\author{
Cihan Akgul Ozmen ${ }^{1}$, Deniz Gokalp ${ }^{2}$, \\ Hasan Nazaroglu ${ }^{1}$, Ayfer Gozu Pirinccioglu ${ }^{3}$ \\ ${ }^{1}$ Department of Radiology, Dicle University School of Medicine, Diyarbakir, Turkey \\ ${ }^{2}$ Department of Endocrinology, Dicle University School of Medicine, Diyarbakir, Turkey \\ ${ }^{3}$ Department of Pediatrics, Dicle University School of Medicine, Diyarbakir, Turkey \\ Email: cihanakgul@gmail.com
}

Received April 30, 2013; revised June 2, 2013; accepted July 1, 2013

Copyright (c) 2013 Cihan Akgul Ozmen et al. This is an open access article distributed under the Creative Commons Attribution License, which permits unrestricted use, distribution, and reproduction in any medium, provided the original work is properly cited.

\begin{abstract}
Objective: The higher risk and fatality of cardiovascular disease in subjects with homozygous familial hypercholesterolemia warrants early screening. Computed tomography angiography is the most promising method at present as a reliable and reproducible noninvasive diagnostic test of cardiovascular disease. We aimed to describe the 64-slice multidetector computed tomography coronary angiography findings of new cases with homozygous familial hypercholesterolemia. Methods: Four patients with homozygous familial hypercholesterolemia (2 female and 2 male) with mean age of $23 \pm 9$ years were included. Mean plasma total and LDL cholesterol were $654 \pm 75 \mathrm{mg} / \mathrm{dl} \mathrm{and} 609 \pm 76 \mathrm{mg} / \mathrm{dl}$. Tomography examinations were performed using a 64-row MDCT-CA system (Brilliance Computed Tomography scanner, Philips Healthcare). Results: All patients presented calcified and mixed atherosclerotic plaques in the ascending aorta near the origin of the coronary arteries. More extensive atherosclerosis was found in Case 1 and Case 2 than in other cases. Case 3 has mild atherosclerosis and Case 4 had normal coronaries but plaques in aorta. In addition, we detected myocardial bridging in left anterior descending artery of 2 cases, calcified plaques in postero-lateral branch artery, and calcification in non-coronary sinus in others. Conclusion: Multidetector computed tomography coronary angiography is a useful, non-invasive tool for detecting early aortic and coronary atherosclerosis in homozygous familial hypercholesterolemia subjects and may be the choice of imaging for most of such subjects.
\end{abstract}

Keywords: Familial Hyperlipidemia; Cardiovascular Disease; Computed Tomography Angiography

\section{Introduction}

Familial hypercholesterolemia is an autosomal codominant inherited disease characterized by lack of functional receptors for low density lipoproteins (LDL) on cell surface in liver and peripheral tissues; as a result of LDL receptor mutations, Ref. [1] elevated plasma cholesterol levels and premature atherosclerosis. The disease has an approximate heterozygote frequency of 1 in 500, and a homozygote frequency of 1 in l,000,000 in the general population [1]. Atherosclerosis develops initially in the aortic root, causing supravalvular aortic stenosis, and then extends into the coronary ostia. The severity of atherosclerosis is proportional to the extent and duration of elevated plasma LDL levels [2,3]. Patients with homozygous familial hypercholesterolemia (hmzFH) develop cutaneous xanthomas in childhood $[1,4,5]$. Prema- ture accelerated atherosclerotic lesions in coronary and aortic valve in subjects with hmzFH exhibit a significantly increased risk of cardiac death, MI or need of vascular intervention before age of 20 years [1,4-7].

The higher risk and fatality of cardiovascular disease (CVD) in subjects with hmzFH warrants [4-7] early screening of CVD in childhood. Coronary artery calcifycation on electron beam tomography was found to be increased in familial hypercholesterolemia subjects [2,3].

Recently the first results of non-invasive angiography with 64 rows multiple detector row computed tomogramphy in 5 patients with hmzFH for non-invasive early detection of atherosclerosis was published [8]. The objective of this study was to describe the 64-slice multidetector computed tomography coronary angiography (MDCT-CA) findings of new cases with hmzFH. 


\section{Materials and Methods}

\subsection{Patients}

Four patients with hmzFH (2 female and 2 male) were included in the present study. The patients were attending at the Endocrinology Department. All subjects gave informed consent prior to study entry and the study was conducted in accordance with Declaration of Helsinki. All of the following four criteria had to be met for a diagnosis of hmzFH: 1) plasma or serum LDL-cholesterol greater than $10 \mathrm{mmol} / \mathrm{L}$; 2) presence of tendon and cutaneous xanthomas at an early age; 3 ) an autosomal inheritance mode of hypercholesterolemia in the relatives; and 4) the presence of primary hypercholesterolemia in the parents of the index case. Additionally, in order to exclude Apo B100 mutation, presence of this mutation was investigated. All measurements were done at the time of first admission while none of the patients were receiving aphaeresis.

\subsection{Computed Tomography Protocol}

All computed tomography examinations were performed using a 64-row multidetector computed tomography system (Brilliance Computed Tomography scanner, Philips Healthcare). Coronary calcium quantification was performed in all subjects with the acquisition parameters of 2.5 mm section thickness, $120 \mathrm{kV}$, and $55 \mathrm{mAs}$. Then the images of MDCT-CA were acquired following the infusion of non-ionic iodine contrast agent by a power injecttor at a rate of with $4 \mathrm{~mL} / \mathrm{s}$ rate via an 18 gauge peripheral line inserted into an antecubital vein. The scan parameters of angiographic series were: $120 \mathrm{kV}$; 500 - 900 mAs; gantry rotation time, $0.40 \mathrm{~s}$; detector collimation, $64 \times 0.625 \mathrm{~mm}$; slice thickness, $0.9 \mathrm{~mm}$. All the data were transferred to a workstation (Extended Brilliance Workspace, Philips Healthcare). Axial, coronal and sagittal reformatted images, volume rendering and maximum intensity projection images were created using Cardiac Viewer and Cardiac CTA programs at the workstation. Plaque segmentation analysis of coronary vessels was also performed. Reconstruction was performed routinely using a window centered at $75 \%$ of the R-R interval in all patients.

All parametric results were expressed as mean \pm standard deviation and non-parametric values as percentages.

\section{Results}

The mean age of patients was $23 \pm 9$ years. No one had diabetes mellitus, hypertension of a history of smoking. Mean plasma total and LDL cholesterol were $654 \pm 75$ $\mathrm{mg} / \mathrm{dl}$ and $609 \pm 76 \mathrm{mg} / \mathrm{dl}$, respectively. Case 1 and case 2 had known CVD at the time of the MDCT-CA. None was symptomatic. All of them were on aggressive lipid- lowering treatment (diet, statin, ezetimib, and apheresis therapy). Apo-B 100 mutation was not detected in any of the cases. Cases 1 and 2 were siblings and other subjects were unrelated. Before admitting to our clinic, invasive angiography was recommended to case 1 and case 2 . They reject the angiography because one cousin of them was died due to coronary atherosclerotic plaque rupture during coronary angiography.

Characteristics of each patient at the time of diagnosis and before the initiation of treatments are shown in Table 1.

All patients presented calcified and mixed atherosclerotic plaques in the ascending aorta near the origin of the coronary arteries (Figures 1(a) and (b)). All cases had right dominance but Case 4 who had co-dominance of coronary arteries. More extensive atherosclerosis was found in Case 1 and Case 2 than in other cases. Case 3 has mild atherosclerosis and Case 4 had normal coronaries but plaques in aorta. MDCT-CA findings of patients are expressed in Table 2.

In addition we detected a $2.5 \mathrm{~cm}$ segment myocardial bridging in proximal left anterior descending artery in case 1, myocardial bridging in middle left anterior descending artery in Case 3, calcified plaques in posterolateral branch artery in Case 2, and calcification in noncoronary sinus at aortic sinus level in Case 4 who had normal coronaries and aorta. We also detected trifurcation of left main coronary artery in Case 2, but no plaques or stenosis in the ramus intermedius branch. Case 4 underwent liver transplantation 2 years later. All cases were alive in the follow-up period of 2 years.

\section{Discussion}

Although guidelines for screening coronary artery disease in hmzFH are lacking due to low number of studies,

Table 1. Features of the cases.

\begin{tabular}{ccccc}
\hline & Case 1 & Case 2 & Case 3 & Case 4 \\
\hline Age (years) & 30 & 31 & 17 & 14 \\
Sex & Female & Male & Female & Male \\
DM & Absent & Absent & Absent & Absent \\
Smoking & Absent & Absent & Absent & Absent \\
Xantomas & Absent & Absent & Absent & Absent \\
Present & Present & Present & Present \\
Age at first Xantomas & 3 & 4 & 5 & 5 \\
LDL aphaeresis & Present & Present & Present & Present \\
T. Cholesterol (mg/dl) & 691 & 732 & 560 & 634 \\
LDL (mg/dl) & 650 & 686 & 512 & 587 \\
\hline
\end{tabular}


Table 2. MDCT-CA findings of patients.

\begin{tabular}{|c|c|c|c|c|}
\hline & Case 1 (Figure 2) & Case 2 (Figures 3(a), (b)) & Case 3 (Figure 4) & Case 4 \\
\hline Previous coronary artery disease & Present & Present & Not investigated & Not investigated \\
\hline Final coronary artery disease & Present & Present & Present & Absent \\
\hline Ca score & 41 & 303 & 0 & 0 \\
\hline Risk\% & 99 & 100 & NA & NA \\
\hline Left main coronary artery & $\begin{array}{l}50 \% \text { stenosis } \\
\text { calcified plaque }\end{array}$ & $50 \%$ stenosis calcified plaque & Normal & Normal \\
\hline left anterior descending artery & Normal & $\begin{array}{c}\text { Severe stenosis in Prox } \\
\text { Calcified and mixed plaques }\end{array}$ & $\begin{array}{l}\text { non severe stenosis in } \\
\text { Prox Soft plaques }\end{array}$ & Normal \\
\hline Left circumflex artery & Normal & $\begin{array}{l}\text { Severe stenosis in middle } \\
\text { Calcified plaques }\end{array}$ & $\begin{array}{l}\text { non severe stenosis in } \\
\text { Prox Soft plaques }\end{array}$ & Normal \\
\hline Right Coronary Artery & $\begin{array}{l}\text { multiple calcified } \\
\text { and soft plaques }\end{array}$ & $\begin{array}{l}\text { Severe stenosis in Prox due to } \\
\text { Mixed Plaque SS in mid and } \\
\text { distal due to Mixed Plaque, }\end{array}$ & $\begin{array}{l}\text { non severe stenosis in } \\
\text { Prox Soft plaques }\end{array}$ & Normal \\
\hline Plaque in ascendant Aorta & Present & Present & Present & Present \\
\hline Aortic calcified plaque at ostium & Present & Present & Present & Present \\
\hline
\end{tabular}

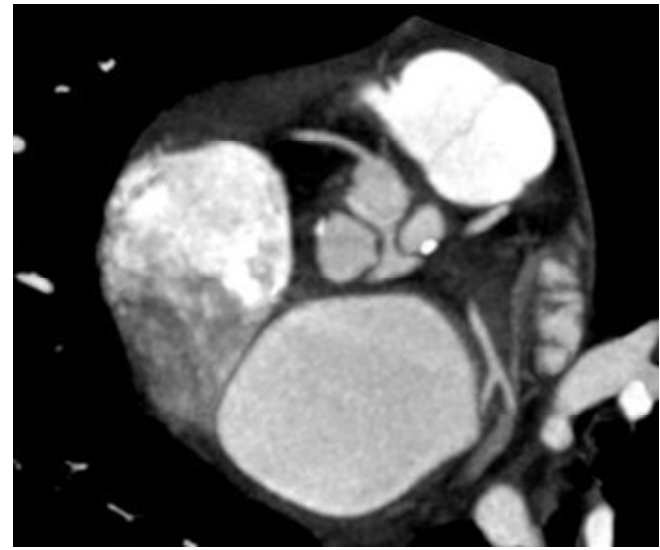

(a)

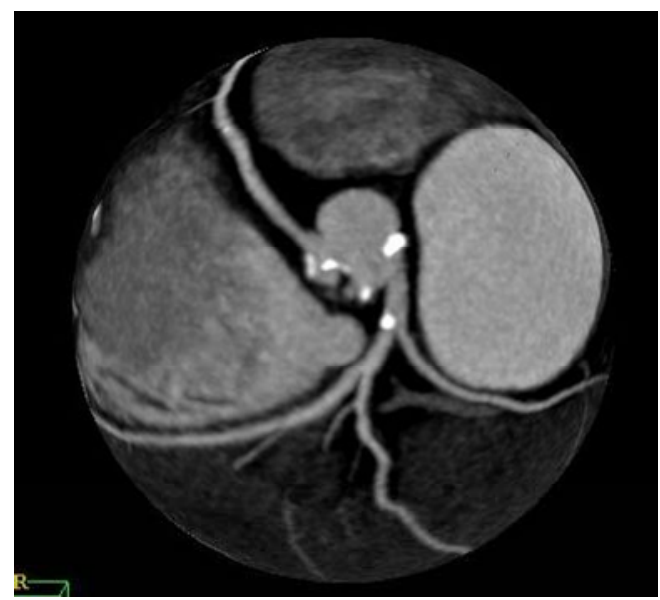

(b)

Figure 1. Scan of Case 4 showed calcified plaques in the left coronary sinus and the non-coronary sinus (a). Extensive calcified plaques in right and left coronary sinus level and calcified plaques in right coronary artery and left main coronary artery on the images of Case 1 (b).

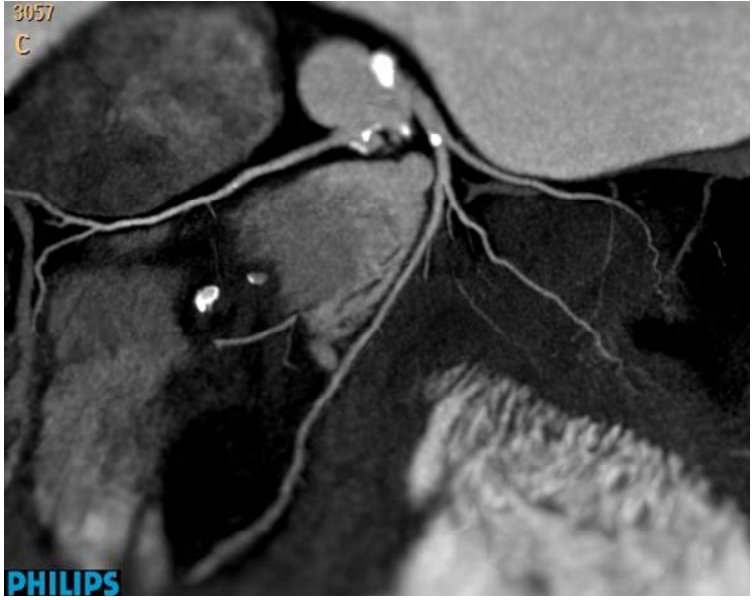

Figure 2. Calcified plaques are visualized in Left main coronary artery of Case 1.

the higher risk and fatality of CVD in subjects with hmzFH warrants [4-7] early and regular screening of CVD in childhood. CVD monitoring at regular intervals using noninvasive methods was recommended [9]. MDCT-CA is the most promising method at present as a reliable and reproducible noninvasive diagnostic test of coronary artery disease [10]. It has a sensitivity of $89 \%$ with a specificity of $96 \%$ and a positive and negative predictive value of $78 \%$ and $98 \%$. The consistently high negative predictive value in all studies suggests that computed tomography angiography will be clinically useful to rule out coronary stenoses in patient suspected coronary artery disease [11].

We found atherosclerosis of coronary arteries in 3 of 4 cases (75\%) and aortic atherosclerosis in all subjects (100\%). All these lesions consisted soft and calcified plaques and had a predominance of involvement of pro- 


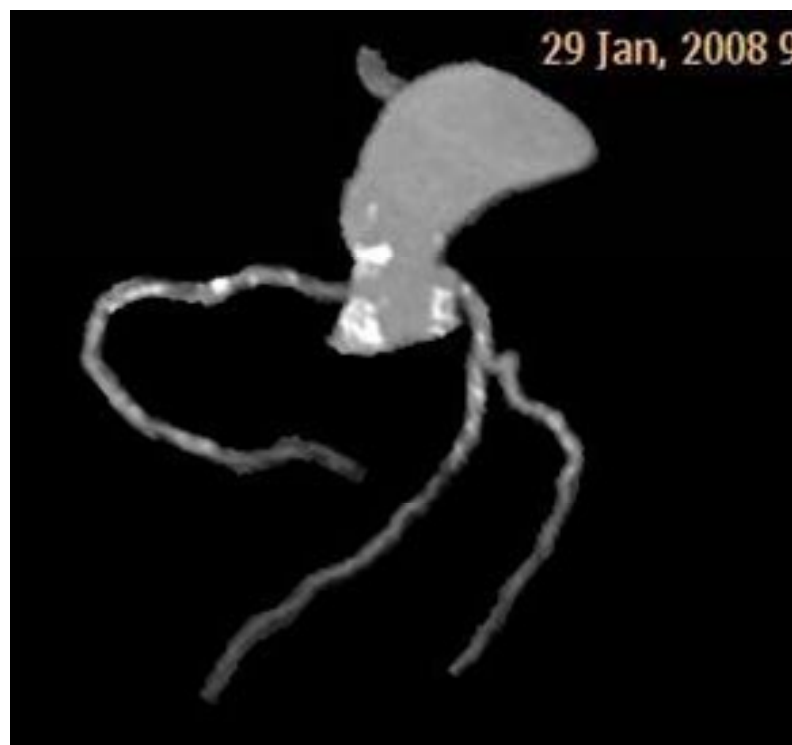

(a)

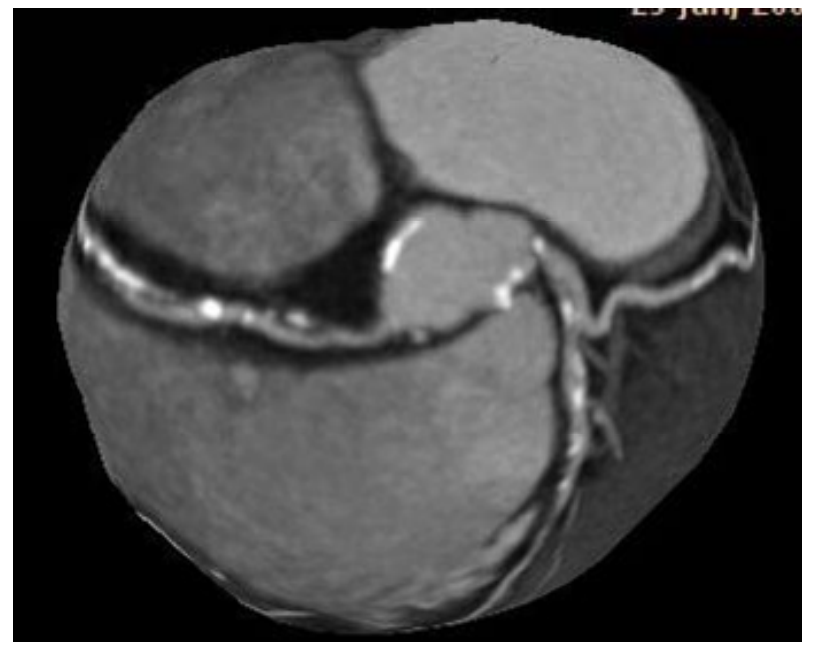

(b)

Figure 3. Calcified plaques in aortic root, the origin of coronary arteries, and all coronary arteries are demonstrated in tree reformatted images of Case 2 (a). Calcified and mixed plaques in the origin of coronary arteries, and all coronary arteries are seen in same patient (b).

ximal segments. Santos et al. [8] also reported severe atherosclerotic plaques in the ascending aorta adjacent to or affecting the coronary artery ostia with MDCT-CA in all five subjects with hmzFH. Four of these cases did not have a diagnosis of CVD and all were asymptomatic with a negative test for ischemia.

Almost all of the patients with angina reported previously had coronary artery disease on angiography, coronary artery disease had been reported even in asymptomatic cases with hmzFH with invasive or computed tomography angiography $[5,8,12]$. Similarly, all of our cases were asymptomatic. Kolansky et al. [12] reported that $50 \%$ of the children aged 7 to 14 years had mild

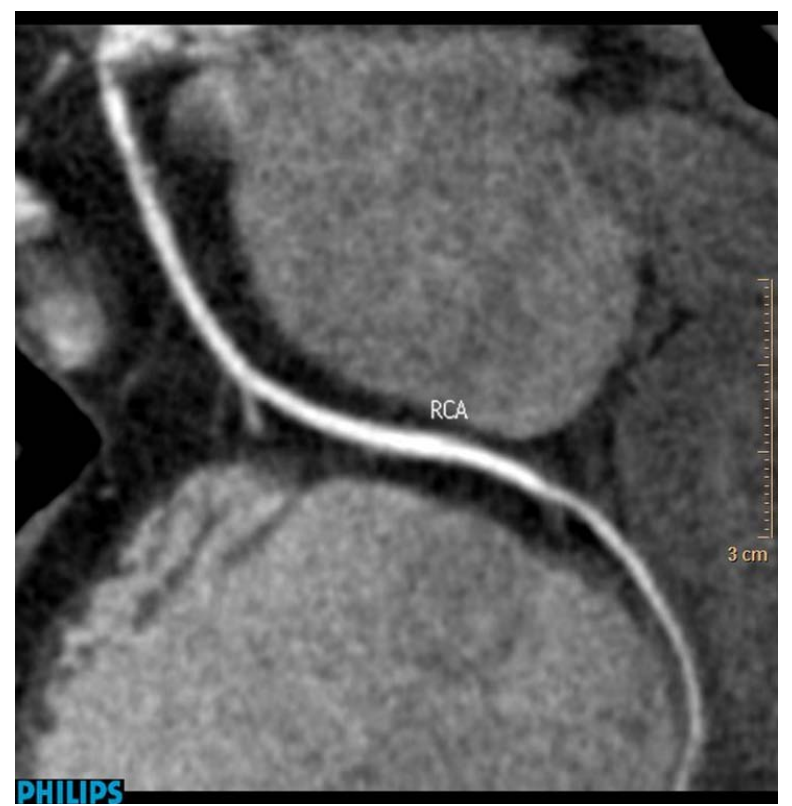

Figure 4. Soft plaques in right coronary artery of Case 3 not yielding severe stenosis.

coronary artery disease with involvement of the left main coronary artery the proximal right coronary artery. In addition, because the stress tests that are commonly used in diagnosis of coronary artery disease did not predict the coronary status of asymptomatic children [12], angiography remain the main tool to diagnose coronary artery disease in this population. Left main coronary artery involvement was present in 2 cases in our study.

The assessment of plaque size and composition may have significant clinical implications in coronary artery disease. MDCT-CA may help identify and characterize non-calcified plaques [10] which are more unstable and prone to acute rupture leading to acute myocardial infarction or acute coronary syndrome than calcified plaques [10].

Occurrence of half of the first coronary events (including sudden cardiac death) in asymptomatic persons [13] and reports of sudden death as the most common reasons of death in hmzFH [6] may be attributed to the plaques that were clearly showed in our patients. Soft plaques were present widely in coronary arteries of Case 1,2 , and 3 . One analysis of 100 patients who were followed for 16 months after coronary computed tomography angiography also demonstrated a higher cardiovascular event rate in patients with non-obstructive plaque detected by multidetector computed tomography [14]. Because of arterial remodelling, plaque progression is not generally reflected in luminal dimensions [10] and computed Tomography imaging goes beyond the delineation of the coronary lumen as provided by invasive angiography [15] which may be advantages of MDCTCA of detecting in asymptomatic cases of hmzFH. 
Therefore invasive angiography may not report soft plaques not affecting the lumen which may the case for Case 3 in our study that had soft plaques in left anterior descending artery, left circumflex artery, and right coronary artery without luminal narrowing. MDCT-CA was also able to demonstrate calcified plaques in coronary sinus or non-coronary sinus which cannot be seen on invasive angiography, as was the case in Case 4.

Prevalence of coronary artery disease was $88 \%$ in cases of hmzFH older than 16 years $9 \%$ in those younger than 16 years [12]. No abnormalities in either coronary angiography were found in the 6 children who were $<6$ years in the cohort consisting 22 children below 16 years of age with hmzFH. Only Case 4 was younger than 16 years of age in our study and had normal coronary arteries.

Invasive coronary angiography was recommended for those with symptoms or signs of ischaemia, because of the risk associated with catheterization of patients with coronary ostial stenosis [9]. Two of our cases refused the angiography because one cousin of them died due to coronary atherosclerotic plaque rupture during coronary angiography in which we were able demonstrate presence and severity of coronary artery disease. Fear of needle may be more important in younger cases of hmzFH who are the candidates of screening.

Because of the three-dimensional nature of the data set, MDCT-CA is very well suited to detect and define the anatomic course of coronary artery anomalies and their relationship to other cardiac and non-cardiac structures [11]. We detected a myocardial bridging of left anterior descending artery in Case 1 and Case 3, as additional findings of MDCT-CA.

Although the ability of separating disease of the wall from disease of the lumen, non-invasive nature, determining the plaque content would represent a great potential of MDCT-CA imaging, radiation exposure and contrast agent doses are comparable with invasive angiography. Needing stable heart rate may make coronary tomography angiography unsuitable in cases with arrhythmia. However, MDCT-CA imaging has the disadvantage of not allowing intervention in comparison to invasive angiography.

Our study has several limitations: First, we were not able to compare our findings with invasive angiography or intravascular ultrasound. The second, the clinical value of MDCT-CA in management of subjects with hmzFH was not evaluated. Our patients were on aggressive lipid-lowering treatment. Lastly, we did not have data on LDL receptor status (receptor-negative or receptor defective) which have significant affects on severity of the disease and its complications [12].

MDCT-CA is a useful, non-invasive tool for detecting early aortic and coronary atherosclerosis in hmzFH sub- jects and may be the choice of imaging for most of such subjects. Results of MDCT-CA may be useful in guiding revascularization strategies and intensifying the cholesterol treatment. Guidelines to screen coronary artery disease in hmzFH and more data on MDCT-CA of subjects with hmzFH are necessary to cope with fatal outcomes of the disease.

\section{REFERENCES}

[1] D. J. Rader, J. Cohen and H. H. Hobbs, "Monogenic Hypercholesterolemia: New Insights in Pathogenesis and Treatment," Journal of Clinical Investigation, Vol. 111, No. 12, 2003, pp. 1795-1803.

[2] J. M. Hoeg, I. M. Feuerstein and E. E. Tucker, "Detection and Quantitation of Calcific Atherosclerosis by Ultrafast Computed Tomography in Children and Young Adults with Homozygous Familial Hypercholesterolemia,” Arteriosclerosis, Thrombosis, and Vascular Biology, Vol. 14, No. 7, 1994, pp. 1066-1074. doi:10.1161/01.ATV.14.7.1066

[3] H. H. Schmidt, S. Hill, E. V. Makariou, I. M. Feuerstein, K. A. Dugi and J. M. Hoeg, "Relation of Choles- terolYear Score to Severity of Calcific Atherosclerosis and Tissue Deposition in Homozygous Familial Hypercholesterolemia," American Journal of Cardiology, Vol. 77, No. 8, 1996, pp. 575-580.

doi:10.1016/S0002-9149(97)89309-5

[4] D. L. Sprecher, E. J. Schaefer, K. M. Kent, R. E. Gregg, L. A. Zech, J. M. Hoeg, et al., "Cardiovascular Features of Homozygous Familial Hypercholesterolemia: Analysis of 16 Patients," American Journal of Cardiology, Vol. 54, No. 1, 1984, pp. 20-30. doi:10.1016/0002-9149(84)90298-4

[5] G. J. Brook, S. Keidar, M. Boulos, E. Grenadier, A. Wiener, N. Shehada, et al., "Familial Homozygous Hypercholesterolemia: Clinical and Cardiovascular Features in 18 Patients," Clinical Cardiology, Vol. 12, No. 6, 1989, pp. 333-338. doi:10.1002/clc.4960120608

[6] H. Mabuchi, S. Miyamoto, K. Ueda, M. Oota, T. Takegoshi, T. Wakasugi, et al., "Causes of Death in Patients with Familial Hypercholesterolemia," Atherosclerosis, Vol. 61, No. 1, 1986, pp. 1-6. doi:10.1016/0021-9150(86)90107-3

[7] H. Mabuchi, J. Koizumi, M. Shimizu and R. Takeda, "Development of Coronary Heart Disease in Familial Hypercholesterolemia,” Circulation, Vol. 79, No. 2, 1989, pp. 225-232. doi:10.1161/01.CIR.79.2.225

[8] R. D. Santos, M. H. Miname, L. R. Martinez, C. E. Rochitte, A. P. Chacra, E. R. Nakandakare, et al., "Non-Invasive Detection of Aortic and Coronary Atherosclerosis in Homozygous Familial Hypercholesterolemia by 64 Slice Multi-Detector Row Computed Tomography Angiography,” Atherosclerosis, Vol. 197, No. 2, 2008, pp. 910-915. doi:10.1016/j.atherosclerosis.2007.08.017

[9] R. P. Naoumova, G. R. Thompson and A. K. Soutar, "Current Management of Severe Homozygous Hypercholesterolaemias,” Current Opinion in Lipidology, Vol. 
15, No. 4, 2004, pp. 413-422. doi:10.1097/01.mol.0000137222.23784.2a

[10] N. E. Manghat, G. J. Morgan-Hughes, A. J. Marshall, C. A. Roobottom, "Multi-Detector Row Computed Tomography: Imaging the Coronary Arteries," Clinical Cardiology, Vol. 60, No. 9, 2005, pp. 939-952. doi:10.1016/j.crad.2005.05.006

[11] S. Schroeder, S. Achenbach, F. Bengel, C. Burgstahler, F. Cademartiri, P. de Feyter, et al., "Cardiac Computed Tomography: Indications, Applications, Limitations, and Training Requirements: Report of a Writing Group deployed by the Working Group Nuclear Cardiology and Cardiac CT of the European Society of Cardiology and the European Council of Nuclear Cardiology,” European Heart Journal, 2008, Vol. 29, No. 4, pp. 531-556. doi:10.1093/eurheartj/ehm544

[12] D. M. Kolansky, M. Cuchel, B. J. Clark, S. Paridon, B. W. McCrindle, S. E. Wiegers, et al., "Longitudinal Evaluation and Assessment of Cardiovascular Disease in Patients with Homozygous Familial Hypercholesterolemia," American Journal of Cardiology, Vol. 102, No. 11, 2008, pp. 1438-1443. doi:10.1016/j.amjcard.2008.07.035

[13] R. A. O’Rourke, B. H. Brundage, V. F. Froelicher, P. Greenland, S. M. Grundy, R. Hachavovitch, et al., "American College of Cardiology/American Heart Association Expert Consensus Document on Electron-Beam Computed Tomography for the Diagnosis and Prognosis of Coronary Artery Disease," Journal of the American College of Cardiology, Vol. 36, No. 1, 2000, pp. 326-340. doi:10.1016/S0735-1097(00)00831-7

[14] G. Pundziute, J. D. Schuijf, J. W. Jukema, E. Boersma, A. de Roos, E. E. van der Wall, et al., "Prognostic Value of Multislice Computed Tomography Coronary Angiography in Patients with Known or Suspected Coronary Artery Disease," Journal of the American College of Cardiology, Vol. 49, No. 1, 2007, pp. 62-70. doi:10.1016/j.jacc.2006.07.070

[15] M. H. Hoffmann and J. Lessick, "Multidetector-Row Computed Tomography for Noninvasive Coronary Imaging,” Expert Review of Cardiovascular Therapy, Vol. 4, No. 4, 2006, pp. 583-594. doi:10.1586/14779072.4.4.583 